\title{
Downregulation of mitochondrial UQCRB inhibits cancer stem cell-like properties in glioblastoma
}

\author{
NARAE JUNG ${ }^{1}$, HO JEONG KWON ${ }^{2}$ and HYE JIN JUNG ${ }^{1}$ \\ ${ }^{1}$ Department of BT-Convergent Pharmaceutical Engineering, Sun Moon University, Tangjeong-myeon, \\ Asan-si, Chungnam 336-708; ${ }^{2}$ Department of Biotechnology, Yonsei University, \\ Seodaemun-gu, Seoul 120-749, Republic of Korea
}

Received September 8, 2017; Accepted October 10, 2017

DOI: $10.3892 /$ ijo.2017.4191

\begin{abstract}
Glioblastoma stem cell targeted therapies have become a powerful strategy for the treatment of this deadliest brain tumor. We demonstrate for the first time that downregulation of mitochondrial ubiquinol-cytochrome $c$ reductase binding protein (UQCRB) inhibits the cancer stem cell-like properties in human glioblastoma cells. The synthetic small molecules targeting UQCRB significantly suppressed not only the self-renewal capacity such as growth and neurosphere formation, but also the metastatic potential such as migration and invasion of glioblastoma stem-like cells (GSCs) derived from U87MG and U373MG at subtoxic concentrations. Notably, the UQCRB inhibitors repressed c-Met-mediated downstream signal transduction and hypoxia-inducible factor-1 $\alpha$ (HIF-1 $\alpha$ ) activation, thereby reducing the expression levels of GSC markers including CD133, Nanog, Oct4 and Sox 2 in the GSCs. Furthermore, the UQCRB inhibitors decreased mitochondrial ROS generation and mitochondrial membrane potential in the GSCs, indicating that they regulate the mitochondrial function in GSCs. Indeed, the knockdown of UQCRB gene by UQCRB siRNA significantly inhibited the cancer stem cell-like phenotypes as well as the expression of stemness markers by blocking mitochondrial ROS/HIF-1 $\alpha /$ c-Met pathway in U87MG GSCs. These findings suggest that UQCRB and its inhibitors could be a new therapeutic target and lead compounds for eliminating cancer stem cells in glioblastoma.
\end{abstract}

\section{Introduction}

Glioblastoma (GBM) is one of the most common and fatal type of brain tumors. Current clinical treatment for GBM

Correspondence to: Professor Hye Jin Jung, Department of BT-Convergent Pharmaceutical Engineering, Sun Moon University, 70 Sunmoon-ro 221, Tangjeong-myeon, Asan-si, Chungnam 336-708, Republic of Korea

E-mail: poka96@sunmoon.ac.kr

Key words: glioblastoma stem cell, UQCRB, mitochondrial ROS, c-Met, HIF-1 $\alpha$ includes maximal surgical resection followed by post-operative radiotherapy and adjuvant chemotherapy (1,2). Despite recent advances in treating other solid tumors, treatment for GBM still remains palliative, with a very poor prognosis and a median survival rate of 12-15 months (3). The best mean survival time with successful tumor resection, radiotherapy and temozolomide (TMZ) chemotherapy may reach up to 18 months (4). The main cause of treatment failure is resistance to radiotherapy and chemotherapy (5). Recent research has applied the cancer stem cell theory of carcinogenesis to tumors, suggesting the existence of a small subpopulation of glioblastoma stem-like cells (GSCs) within GBM (6). GSCs are thought to contribute to tumor progression, treatment resistance and tumor recurrence, and therefore targeting GSCs has emerged as a powerful GBM treatment strategy $(7,8)$. Accumulating evidence has revealed that several molecular markers, such as Wnt, Hedgehog, Notch, transforming growth factor- $\beta$ (TGF- $\beta$ ), epidermal growth factor receptor (EGFR), telomerase and efflux transporters, are important for self-renewal and differentiation of GSCs $(9,10)$. Although they might be useful for targeted therapy in GSCs, finding novel therapeutic targets and agents to eradicate GSCs can provide a promising treatment strategy that significantly improves GBM patient survival and quality of life.

In a previous study, terpestacin from fungal metabolites was discovered as a new angiogenesis inhibitor with a unique bicyclo sesterterpene structure (11). The deconvolution of its cognate target protein using a target identification method has revealed that terpestacin binds to the ubiquinol-cytochrome $c$ reductase binding protein (UQCRB) of complex III in mitochondrial respiratory chain (12). Terpestacin binding to UQCRB resulted in inhibition of hypoxia-induced ROS generation and such inhibition blocked hypoxia-inducible factor (HIF) activation and tumor angiogenesis in vivo (12). Furthermore, UQCRB-mediated mitochondrial ROS played a critical role in hypoxic signaling in tumor cells as well as vascular endothelial growth factor (VEGF) signaling in endothelial cells (13). Therefore, UQCRB has become a new therapeutic target for anti-angiogenic and antitumor drug development. More recently, based on a target-based screen with structural information on the binding mode of terpestacin and UQCRB, novel synthetic small molecules targeting UQCRB were developed $(14,15)$. The compounds specifically 
bound to UQCRB and inhibited mitochondrial ROS-mediated hypoxic signaling, resulting in potent suppressive effects against key angiogenic processes of endothelial cells activated by VEGF in vitro as well as antiangiogenic and antitumor activities in vivo, without inducing cytotoxicity $(14,15)$.

In the present study, to explore a new therapeutic strategy targeting GSCs by regulation of mitochondrial function, the effects of mitochondrial UQCRB inhibitors against GSCs were investigated. Our results showed that the UQCRB inhibitors effectively suppressed the self-renewal capacity and metastatic potential of GSCs at subtoxic concentrations. Particularly, the inhibitory action of the UQCRB inhibitors against GSCs was associated with blocking of c-Met signaling pathways and subsequent reduction of the expression levels of GSC markers, through the regulation of mitochondrial function in GSCs. In addition, UQCRB depletion in GSCs phenocopied all the effects of the UQCRB inhibitors, suggesting that UQCRB and its inhibitors could be a new therapeutic target and lead compounds for eliminating cancer stem cells in GBM.

\section{Materials and methods}

Materials. UQCRB inhibitors, 1c (A1893) and 1f (A1938), were synthesized and characterized as described previously (15). The compounds were dissolved in dimethyl sulfoxide (DMSO) at a concentration of $100 \mathrm{mM}$ as a stock solution and then further diluted with culture media for appropriate working doses. The negative control groups were treated with equal volumes of DMSO. Gelatin and laminin were purchased from Sigma-Aldrich (St. Louis, MO, USA), and Matrigel ${ }^{\circledR}$ was obtained from BD Biosciences (San Jose, CA, USA). Anti-CD133 and anti-HIF-1 $\alpha$ antibodies were purchased from Miltenyi Biotec GmbH (Bergisch Gladbach, Germany) and BD Biosciences, respectively. Anti-Nanog, anti-Sox2, anti-Oct4, anti-phospho-Met, anti-Met, anti-phospho-Stat3, anti-Stat3, anti-phospho-Akt, anti-Akt, anti-phospho-Erk1/2, anti-Erk1/2, anti-VEGF and anti- $\beta$-actin antibodies were obtained from Cell Signaling Technology (Danvers, MA, USA).

Cell culture. Human glioblastoma cell lines, U87MG and U373MG, were obtained from the Korean Cell Line Bank (KCLB). The cells were cultured in minimum essential medium (Gibco, Grand Island, NY, USA) supplemented with $10 \%$ fetal bovine serum (Gibco) and $1 \%$ penicillin-streptomycin-amphotericin B (Lonza, Walkersville, MD, USA), and maintained at $37^{\circ} \mathrm{C}$ in a humidified $5 \% \mathrm{CO}_{2}$ incubator. To enrich glioblastoma stem-like cells (GSCs), the cells grown in serum-based media were suspended in Dulbecco's modified Eagle's medium/ Nutrient Mixture F-12 (Gibco) containing 1X B-27 serum-free supplement (Gibco), $5 \mu \mathrm{g} / \mathrm{ml}$ heparin (Sigma-Aldrich), $2 \mathrm{mM}$ L-glutamine (Gibco), $20 \mathrm{ng} / \mathrm{ml}$ epidermal growth factor (EGF; Gibco), $20 \mathrm{ng} / \mathrm{ml}$ basic fibroblast growth factor (bFGF; Koma Biotech, Seoul, Korea) and 1\% penicillin/streptomycin (Gibco). The serum-free media with EGF and bFGF were added to the cells twice a week. Neurospheres were passaged every 7 days by dissociating with Accutase (Millipore, Temecula, CA, USA).

Cell growth assay. Cell growth was evaluated by WST-1 assay, a water-soluble tetrazolium salt method. Neurosphere cells were dissociated with Accutase and seeded into 96-well culture plate at a density of $3 \times 10^{3}$ cells/well using the serum-free media with EGF and bFGF. After 7 days of exposure to UQCRB inhibitors, $10 \mu$ WST-1 reagent solution (Dogen, Korea) was added to each well, and the cells were incubated for additional $3 \mathrm{~h}$ at $37^{\circ} \mathrm{C}$. The absorbance was measured at a wavelength of $450 \mathrm{~nm}$ using a microplate reader (Thermo Scientific, Vantaa, Finland).

Cell viability assay. Cell viability was evaluated by trypan blue exclusion assay. Dissociated neurosphere cells were seeded at a density of $1 \times 10^{5}$ cells/well in 12-well culture plate using the serum-free media with EGF and bFGF. UQCRB inhibitors were added to each well and the cells were incubated for up to 7 days. The cells were stained with trypan blue and counted using a hemocytometer. Cell viability was calculated as the number of viable cells divided by the total number of cells.

Neurosphere formation assay. Neurosphere cells were dissociated with Accutase, and seeded into 96-well culture plate at a density of 50 cells per well using the serum-free media with EGF and bFGF. The cells were treated with UQCRB inhibitors and cultured for 1-2 weeks. The number of neurospheres in each well was counted under an optical microscope (Olympus, Center Valley, PA, USA). Data were presented as the percentage of sphere-forming cells relative to DMSO-treated control.

Migration assay. The ibidi culture inserts (ibidi $\mathrm{GmbH}$, Martinsried, Germany), which consist of two chambers separated by a $500-\mu \mathrm{m}$ divider, were used for the migration assay. The inserts were placed into a laminin-coated 24-well culture plate using sterile tweezers. Neurosphere cells were dissociated with Accutase, and single-cell suspensions were prepared at a density of $5 \times 10^{5}$ cells $/ \mathrm{ml}$, of which $70 \mu \mathrm{l}$ was transferred to each chamber. After cell attachment for $24 \mathrm{~h}$, the culture inserts were removed by using sterile tweezers. The cells were washed with phosphate-buffered saline (PBS) to remove detached cells and incubated with the GSC culture media in the absence or presence of UQCRB inhibitors for up to $48 \mathrm{~h}$. The perimeter of the central cell-free zone was confirmed under an optical microscope (Olympus).

Invasion assay. Cell invasion was assayed using a Transwell ${ }^{\circledR}$ chamber system with polycarbonate filter inserts with a pore size of $8.0 \mu \mathrm{m}$ (Corning Costar, Acton, MA, USA). The lower side of the filter was coated with $10 \mu \mathrm{l}$ gelatin $(1 \mathrm{mg} / \mathrm{ml})$ and the upper side was coated with $10 \mu 1$ Matrigel $(3 \mathrm{mg} / \mathrm{ml})$. The GSCs $\left(2 \times 10^{5}\right)$ were placed in the upper chamber of the filter, and UQCRB inhibitors were added to the lower chamber filled with the serum-free media containing EGF and bFGF. The chamber was incubated at $37^{\circ} \mathrm{C}$ for $48 \mathrm{~h}$, and the cells were subsequently fixed with methanol and stained with hematoxylin/eosin. The total number of cells that invaded the lower chamber of the filter was counted using an optical microscope (Olympus).

Western blot analysis. Cell lysates were separated by $10 \%$ SDS-PAGE electrophoresis and the separated proteins 
were transferred to polyvinylidene difluoride membranes (Millipore, Billerica, MA, USA) using standard electroblotting procedures. The blots were blocked and immunolabeled with primary antibodies against CD133, Nanog, Sox2, Oct4, phospho-Met (Tyr1234/1235), Met, phospho-Stat3 (Tyr705), Stat3, phospho-Akt (Ser473), Akt, phospho-Erk1/2 (Thr202/Tyr204), Erk1/2, HIF-1 $\alpha$, VEGF and $\beta$-actin overnight at $4^{\circ} \mathrm{C}$. Immunolabeling was detected with an enhanced chemiluminescence (ECL) kit (Bio-Rad, Hercules, CA, USA), according to the manufacturer's instructions.

Measurement of reactive oxygen species (ROS). Intracellular ROS and mitochondrial ROS levels were detected with $2^{\prime}, 7^{\prime}$-dichlorodihydrofluorescein diacetate ( $\left.\mathrm{H}_{2} \mathrm{DCFDA}\right)$ and MitoSOX ${ }^{\mathrm{TM}}$ Red mitochondrial superoxide indicator (Molecular Probes, Eugene, OR, USA), respectively. For the assays, the GSCs seeded at a density of $5 \times 10^{4}$ cells/well in 96-black well culture plate were treated with UQCRB inhibitors for $48 \mathrm{~h}$. After incubation with $\mathrm{H}_{2}$ DCFDA $(10 \mu \mathrm{M})$ or MitoSOX Red $(5 \mu \mathrm{M})$ for $10 \mathrm{~min}$, the fluorescence intensity was detected using a multimode microplate reader (Thermo Scientific) at the excitation/emission wavelengths of $495 / 529 \mathrm{~nm}$ for intracellular ROS and 510/580 nm for mitochondrial ROS, respectively. The fluorescent images were also obtained using an Optinity KI-2000F fluorescence microscope (Korea Lab Tech, Seong Nam, Korea).

Mitochondrial membrane potential determination. The mitochondrial membrane potential was detected using the fluorescent, lipophilic dye, JC-1 (5,5',6,6'-tetrachloro-1,1',3,3'tetraethylbenzimidazol-carbocyanine iodide, Sigma-Aldrich). At hyperpolarized membrane potentials, this dye forms a red fluorescent J-aggregate, whereas at depolarized membrane potentials, this dye remains in its green fluorescent monomeric form. The GSCs were seeded in 24-black well culture plate at a density of $5 \times 10^{4}$ cells/well and treated with UQCRB inhibitors for $48 \mathrm{~h}$. The cells were incubated with JC-1 $(5 \mu \mathrm{M})$ for $20 \mathrm{~min}$ and the images were obtained using an Optinity $\mathrm{KI}-2000 \mathrm{~F}$ fluorescence microscope.

UQCRB silencing. Human UQCRB-specific siRNA (siUQCRB) was constructed as described previously (13). The sense and antisense sequences of this siRNA were 5'-GGG UUA AUG CGA GAU GAU ACA AUA U-3' and 5'-AUA UUG UAU CAU CUC GCA UUA ACC C-3', respectively. For depletion of UQCRB mRNA, U87MG GSCs seeded in laminin-coated culture plate with the GSC culture media were transfected with either scrambled negative siRNA or UQCRB siRNA using Lipofectamine 3000 transfection reagent (Invitrogen, Grand Island, NY, USA) according to the manufacturer's instructions. Interference of UQCRB mRNA was validated through reverse transcriptase-polymerase chain reaction (RT-PCR) analysis using specific primers for UQCRB (sense, 5'-ATGGCTGGTAAGCAGGCC-3'; antisense, 5'-CTTCTTTGCCCATTCTTC-3').

Statistical analysis. The results are expressed as the mean \pm standard error (SE). Student's t-test was used to determine statistical significance between the control and test groups. A P-value of $<0.05$ was considered statistically significant.<smiles>CC(C)(C)c1ccc(S(=O)(=O)Nc2ccc(O)cc2)cc1</smiles><smiles>CC(C)(C)[14CH3]</smiles>

Figure 1. The chemical structures of UQCRB inhibitors.

\section{Results}

The effect of UQCRB inhibitors on the proliferation of GSCs. All established glioma cell lines, like most other cancer cell lines, are grown in media containing serum, whereas GSCs are grown as neurospheres in serum-free media, since serum causes irreversible differentiation of primary tumor cells $(16,17)$. Accordingly, in this study, GSCs were propagated under an optimal condition using serum-free media supplemented with basic FGF and EGF (18). In order to determine the therapeutic effect of mitochondrial UQCRB inhibitors against GSCs, two synthetic small molecules that are known to target UQCRB, 1c (A1893) and 1f (A1938), were analyzed in the present study (15) (Fig. 1).

We first examined the effect of UQCRB inhibitors on the proliferation of GSCs derived from U87MG and U373MG cells using a water-soluble tetrazolium salt method. The UQCRB inhibitors suppressed the proliferation of both GSCs in a dose-dependent manner (Fig. 2A). To further evaluate whether the GSC growth inhibition by UQCRB inhibitors was due to cytotoxic effects, a viability assay was performed using the trypan blue exclusion method. The viability of GSCs exceeded $80 \%$ relative to DMSO-treated control cells, even after treatment with $40 \mu \mathrm{M}$ of UQCRB inhibitors for 7 days (Fig. 2B). Therefore, the UQCRB inhibitors can inhibit the proliferation of U87MG and U373MG GSCs at subtoxic concentrations.

The effect of UQCRB inhibitors on the stemness of GSCs. Sphere-forming assays have been widely used to evaluate the stemness of cells, their capacity for self-renewal and differentiation, both of which are instrumental in cancer cell formation $(6,19)$. As shown in Fig. 3, the neurosphere formation ability of both GSCs was markedly suppressed by treatment with the UQCRB inhibitors. These results indicate that UQCRB inhibitors can reduce the self-renewal capacity of GSCs. 
A

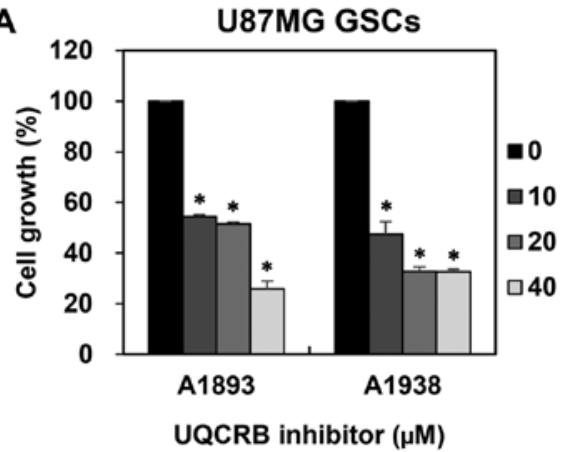

B

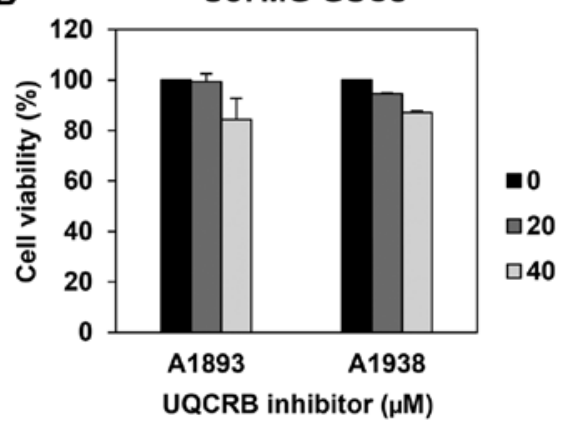

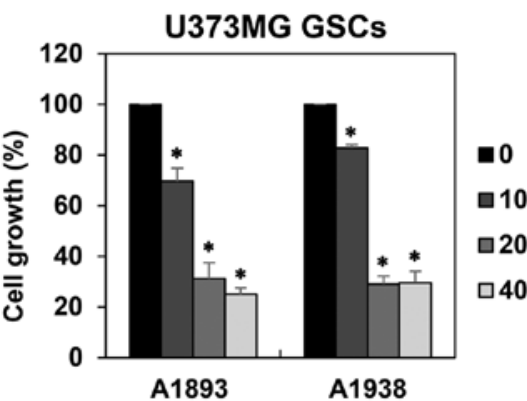

UQCRB inhibitor $(\mu \mathrm{M})$

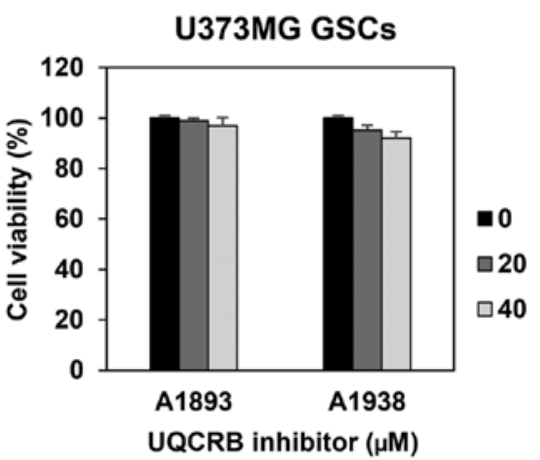

Figure 2. The effect of UQCRB inhibitors on the growth of glioblastoma stem-like cells. (A and B) GSCs from U87MG and U373MG were treated with UQCRB inhibitors for 7 days. (A) Cell growth was measured using the WST-1 colorimetric assay. ${ }^{*} \mathrm{P}<0.05$ versus the control. (B) Cell viability was measured by the trypan blue assay.
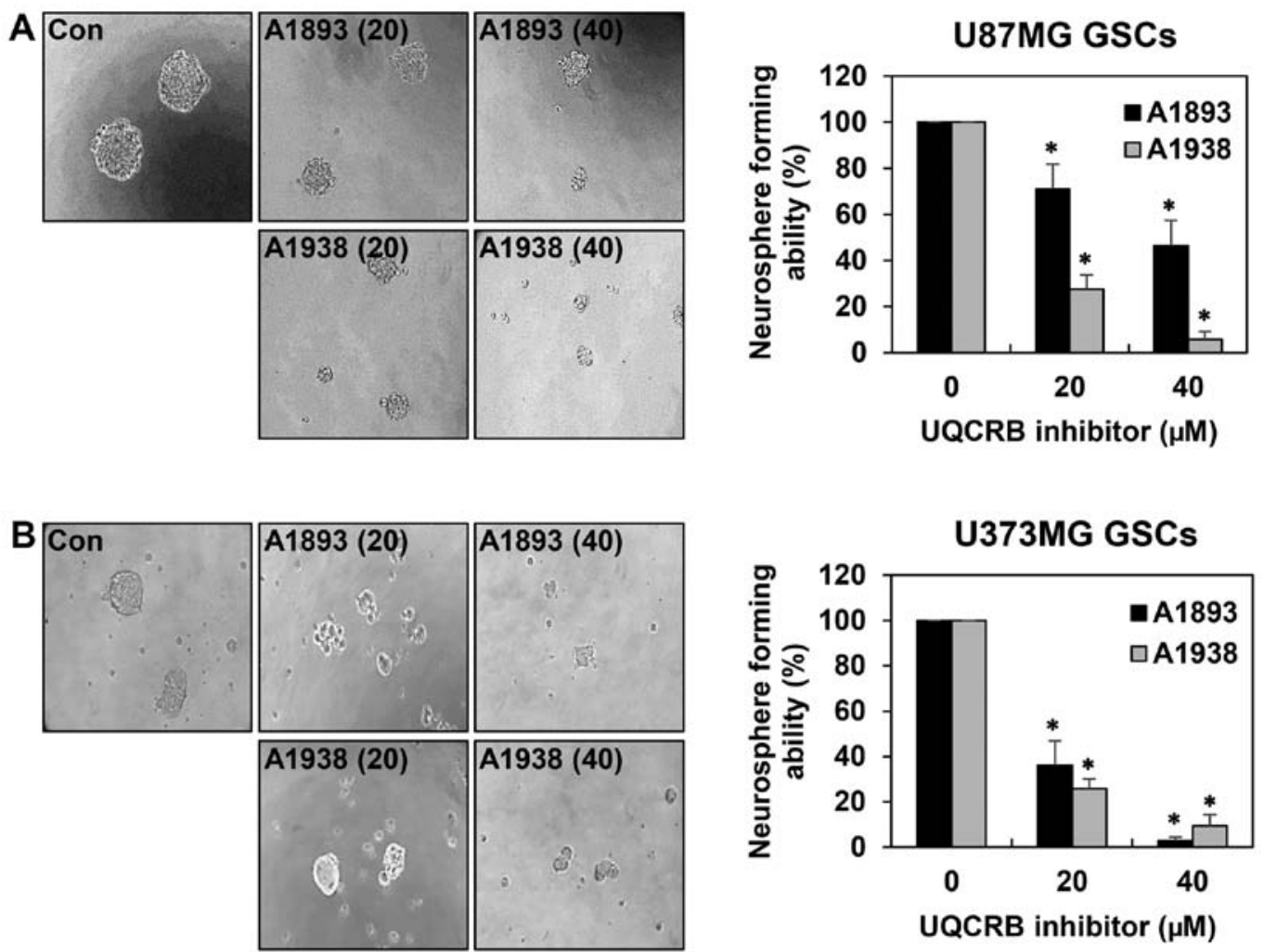

Figure 3. The effect of UQCRB inhibitors on the neurosphere-forming ability of glioblastoma stem-like cells. GSCs derived from (A) U87MG and (B) U373MG were treated with UQCRB inhibitors for 1-2 weeks. The formed neurospheres were observed and then counted under a microscope. " $\mathrm{P}<0.05$ versus the control.

The effect of UQCRB inhibitors on the migration and invasion of GSCs. GSCs have been implicated in accelerating tumor metastasis $(20,21)$. We thus assessed whether UQCRB inhibitors have an effect on the migration and invasion 

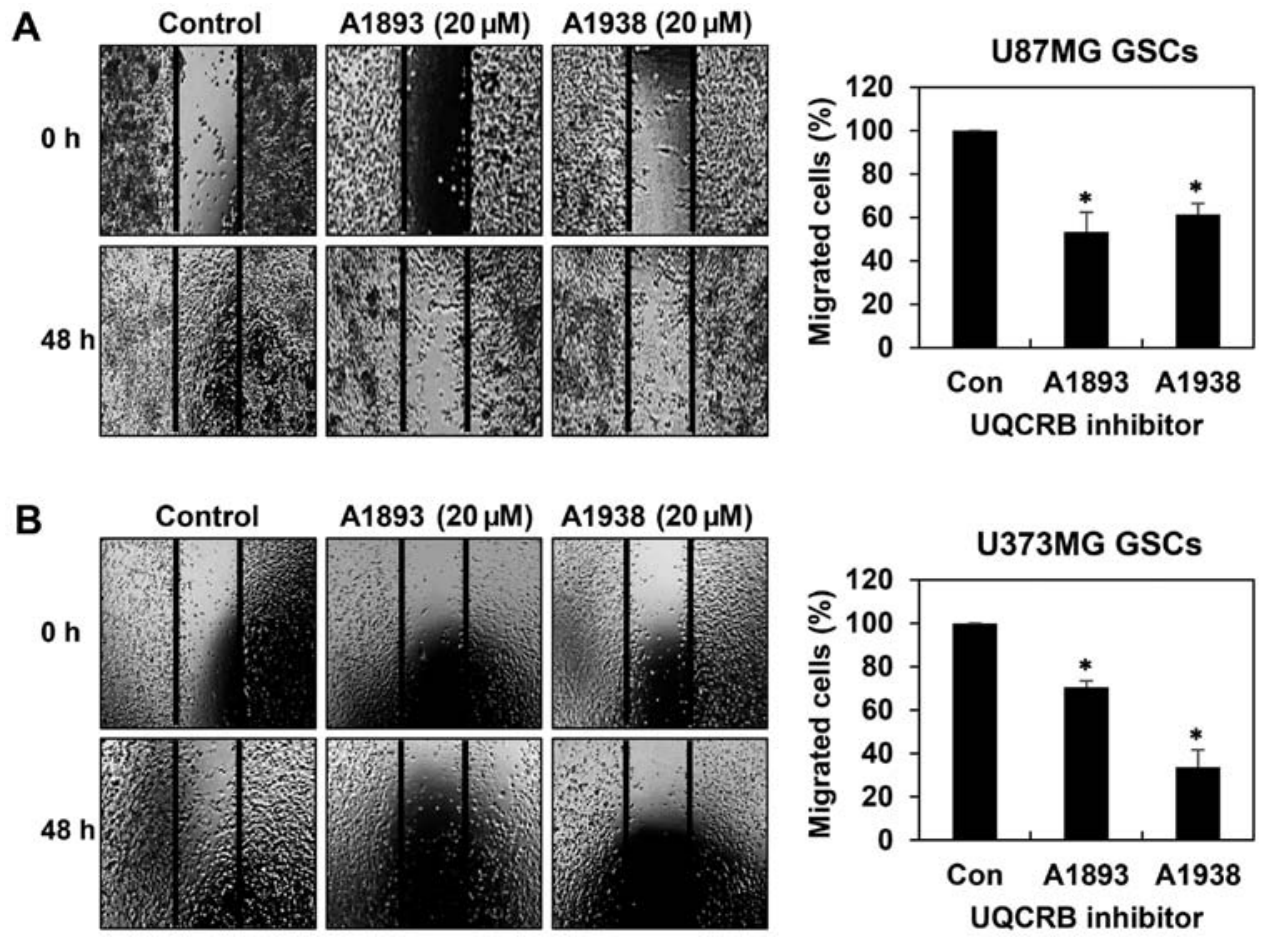

Figure 4. The effect of UQCRB inhibitors on the migration of glioblastoma stem-like cells. GSCs from (A) U87MG and (B) U373MG were seeded into laminin-coated culture plate and incubated with the GSC culture media in the absence or presence of UQCRB inhibitors for $48 \mathrm{~h}$. The cells migrated into the gap were counted under an optical microscope. Black lines indicate the edge of the gap at $0 \mathrm{~h}$. " $\mathrm{P}<0.05$ versus the control.

A
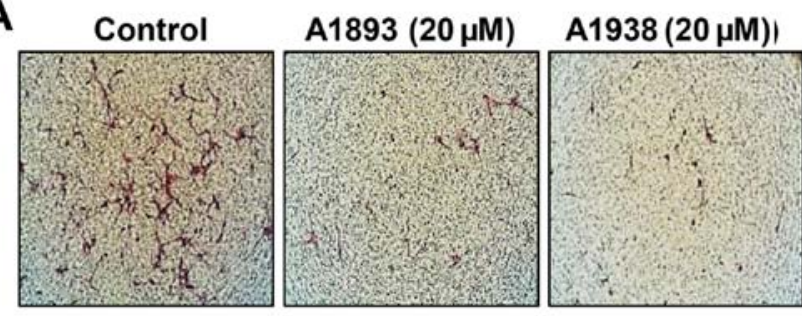

B

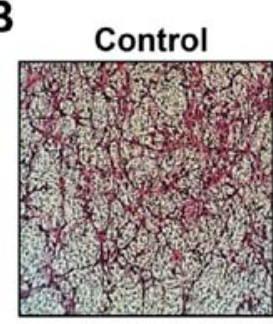

A1893 $(20 \mu \mathrm{M})$

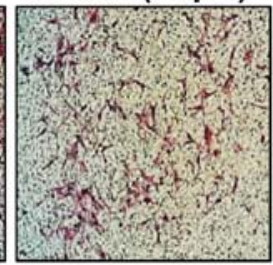

$\mathrm{A} 1938(20 \mu \mathrm{M})$

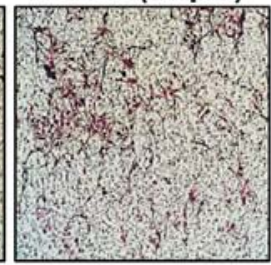

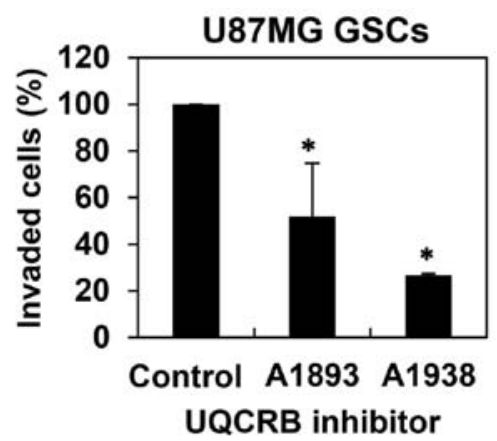

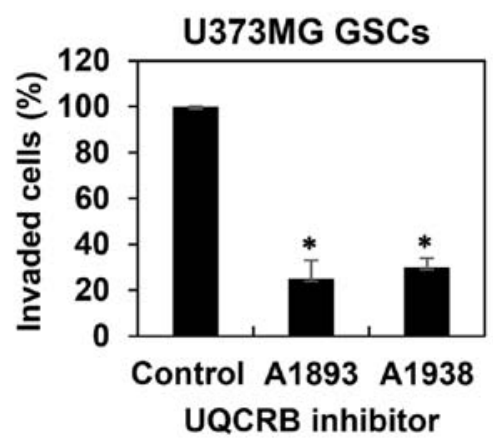

Figure 5. The effect of UQCRB inhibitors on the invasion ability of glioblastoma stem-like cells. The invasiveness of (A) U87MG and (B) U373MG GSCs was analyzed using Matrigel-coated polycarbonate filters. The GSCs were incubated in the absence or presence of UQCRB inhibitors for $48 \mathrm{~h}$. The cells penetrating the filters were stained and counted under an optical microscope. ${ }^{*} \mathrm{P}<0.05$ versus the control.

of GSCs. We first confirmed their effect on the migration of both GSCs in $48 \mathrm{~h}$ after treatment when compared to control conditions using wound healing assay. The UQCRB inhibitors led to significant reduction of cell migration in U87MG and U373MG GSCs (Fig. 4). Next, invasion assay was performed by employing the Matrigel-coated transwell chamber system. The UQCRB inhibitors distinctly decreased the invasion of U87MG and U373MG GSCs when compared with controls (Fig. 5). These data demonstrate that UQCRB inhibitors suppress the metastatic capability of GSCs. 

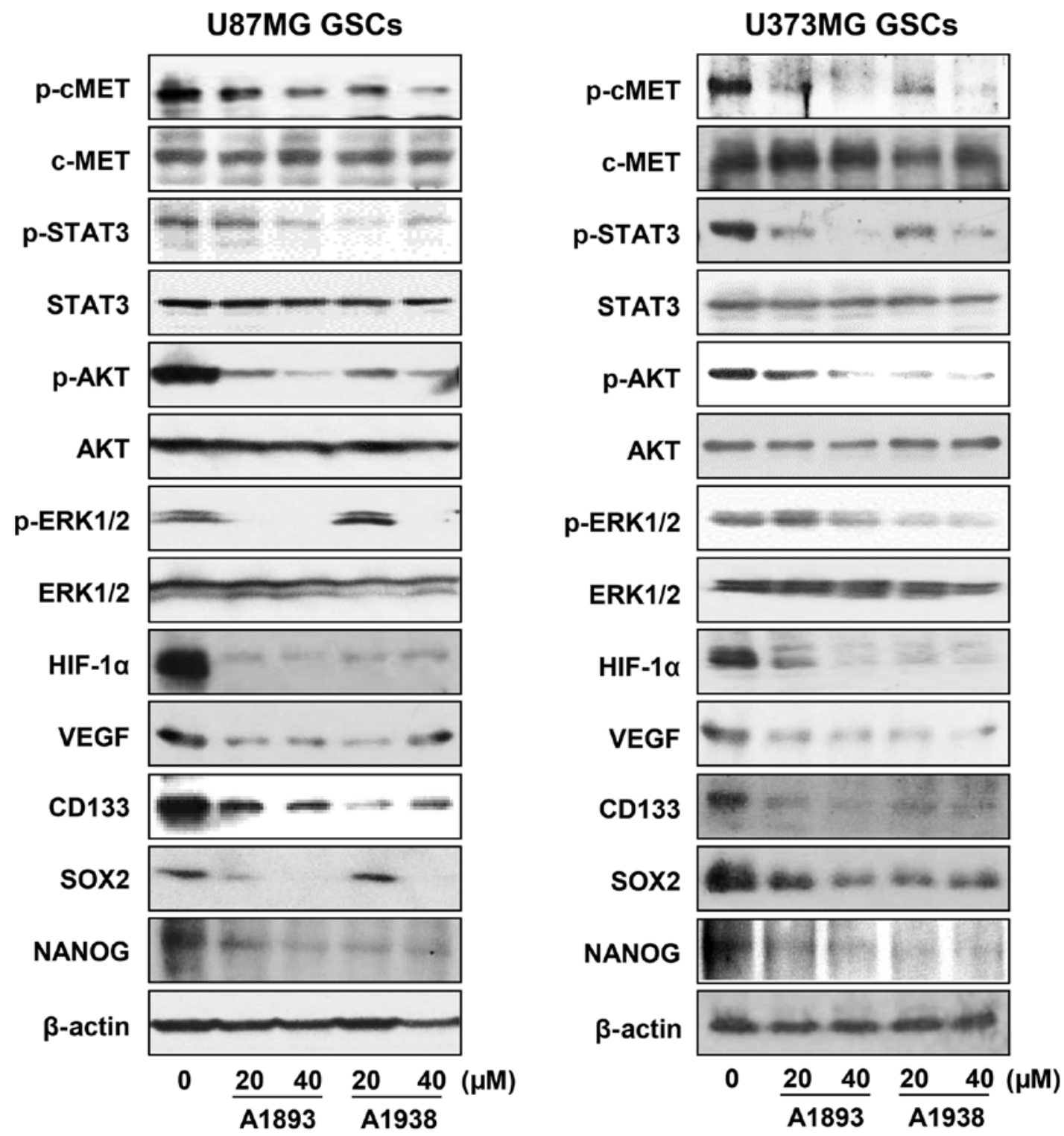

Figure 6. The effect of UQCRB inhibitors on the c-Met signaling, HIF activity and stemness markers in glioblastoma stem-like cells. U87MG and U373MG GSCs were treated with UQCRB inhibitors for $48 \mathrm{~h}$, and the protein levels were detected by western blot analysis using specific antibodies. The levels of $\beta$-actin were used as an internal control.

The effect of UQCRB inhibitors on the c-Met signaling pathway in GSCs. Recent studies have shown that c-Met signaling induces glioma malignancy by increasing GSC population through upregulation of stemness supporting transcription factors such as Sox 2, Klf4, c-Myc, Oct4 and Nanog $(22,23)$. We thus investigated the effect of UQCRB inhibitors on the c-Met signaling cascade in U87MG and U373MG GSCs. Treatment with the UQCRB inhibitors effectively inhibited the phosphorylation of c-Met and its downstream signal transduction effectors including STAT3, Akt and ERK1/2, without affecting the total protein levels, in either GSC (Fig. 6). Recent reports have revealed that hypoxia-inducible factors (HIFs) stimulate specific signaling pathways and transcription factors that control cancer stem cell self-renewal and multipotency and are highly expressed in GSCs $(24,25)$. The UQCRB inhibitors significantly reduced the protein levels of HIF-1 $\alpha$ as well as its transcriptional target gene, VEGF in GSCs (Fig. 6). They also decreased the expression of GBM stemness markers such as CD133, Nanog and Sox2, which are known to be regulated by c-Met and HIFs (Fig. 6). Taken together, these data suggest that the suppressive effect of UQCRB inhibitors on the proliferation, stemness, migration and invasiveness of GSCs might be partly associated with the downregulation of c-Met signaling and HIF activity.

The effect of UQCRB inhibitors on the mitochondrial function in GSCs. Recent studies have indicated that ROS contribute to cancer stem cell-like properties and play pivotal roles in tumorigenesis, metastasis and resistance to radiation and chemotherapy (26-28). Since UQCRB functions as a mitochondrial ROS mediator $(12,13)$, we examined whether UQCRB inhibitors affect intracellular ROS levels in U87MG and U373MG GSCs using a fluorescent probe for ROS measurement, 2',7'-dichlorofluorescein. The UQCRB inhibitors reduced the intracellular ROS levels in both GSCs (Fig. 7A). 
A

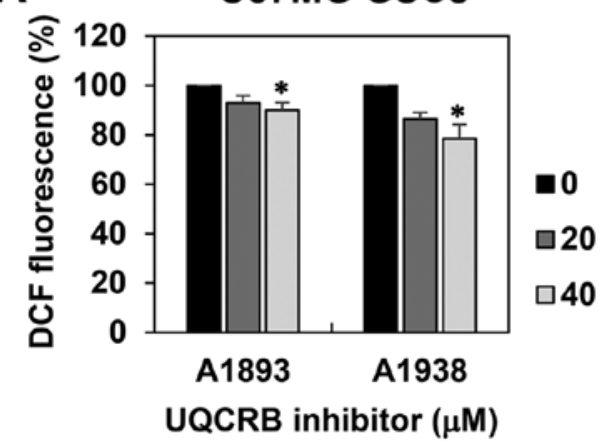

B

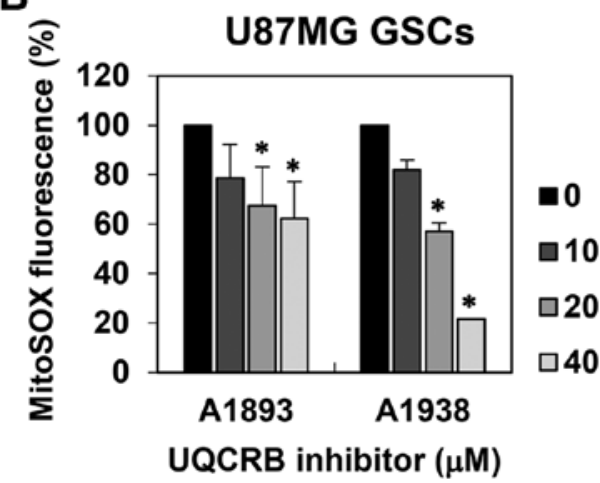

U373MG GSCs
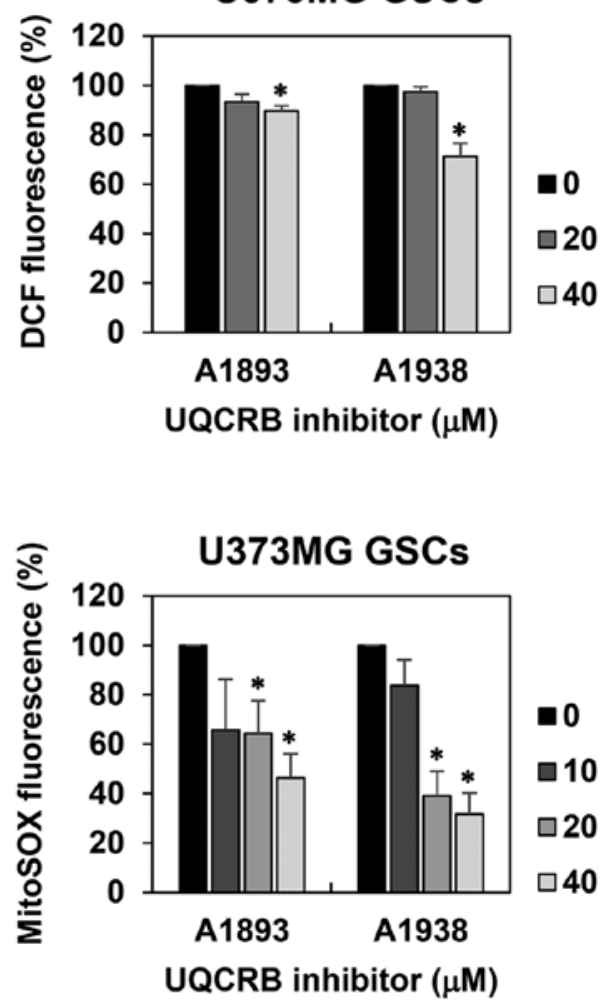

Figure 7. The effect of UQCRB inhibitors on the generation of ROS in glioblastoma stem-like cells. (A and B) GSCs from U87MG and U373MG were treated with UQCRB inhibitors for 48 h. (A) Intracellular ROS levels were determined by the 2',7'-dichlorofluorescein (DCF) fluorescence. (B) Mitochondrial ROS levels were determined by MitoSOX ${ }^{\mathrm{TM}}$ Red fluorescence. ${ }^{*} \mathrm{P}<0.05$ versus the control.

A
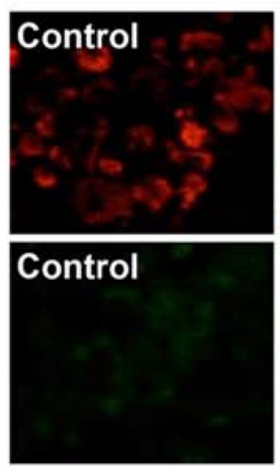

U87MG GSCs

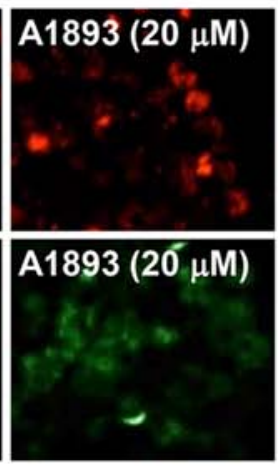

B

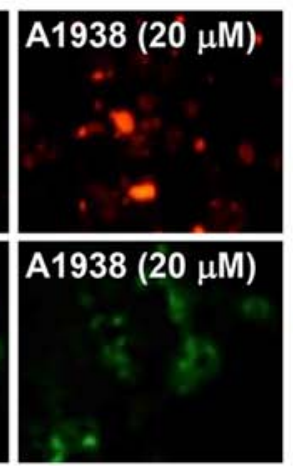

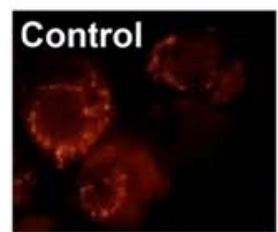

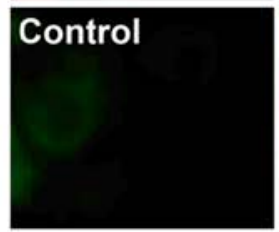

U373MG GSCs

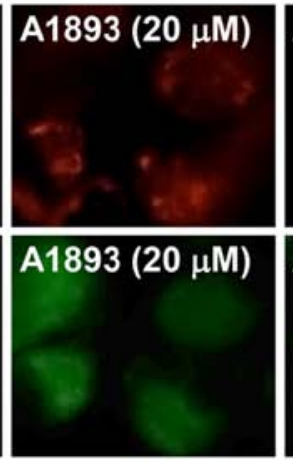

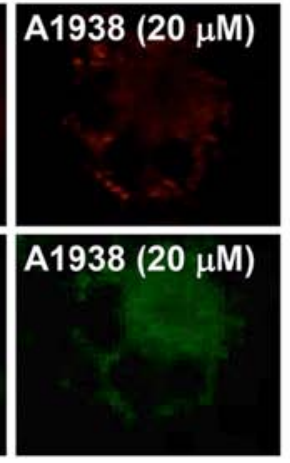

Figure 8. The effect of UQCRB inhibitors on the mitochondrial membrane potential in glioblastoma stem-like cells. (A) U87MG and (B) U373MG GSCs were treated with UQCRB inhibitors for $48 \mathrm{~h}$ and stained with JC-1. Fluorescence images were obtained with a fluorescence microscope.

Moreover, mitochondrial ROS levels in GSCs were measured using a MitoSOX ${ }^{\mathrm{TM}}$ Red mitochondrial superoxide indicator. The UQCRB inhibitors noticeably inhibited the mitochondrial ROS generation in both GSCs (Fig. 7B).

Next, to investigate whether UQCRB inhibitors cause mitochondrial dysfunction in GSCs, the mitochondrial membrane potential was measured using the JC-1 fluorescent marker. Treatment with the UQCRB inhibitors led to a decrease in red fluorescence and an increase in green fluorescence in U87MG and U373MG GSCs, indicating that they cause a loss of the mitochondrial membrane potential in the cells (Fig. 8). These data demonstrate that UQCRB inhibitors may modulate the mitochondrial function in GSCs, especially at mitochondrial ROS generation.

The effect of UQCRB knockdown on the cancer stem cell-like phenotypes of GBM cells. To elucidate the role of UQCRB in the maintenance of GSCs, we performed UQCRB depletion experiments in U87MG GSCs. The cells were transfected with either UQCRB siRNA (siUQCRB) or scrambled negative siRNA, and knockdown of the UQCRB gene was confirmed through RT-PCR analysis (Fig. 9A). We first investigated the effect of UQCRB knockdown on the cancer stem cell-like phenotypes of GBM cells. As shown in Fig. 9B, 
A

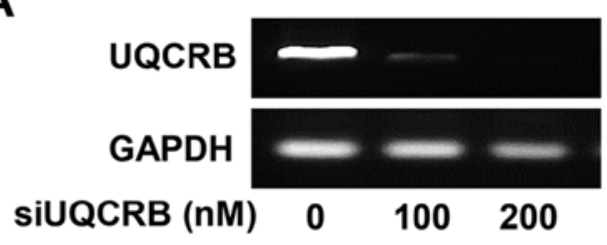

B

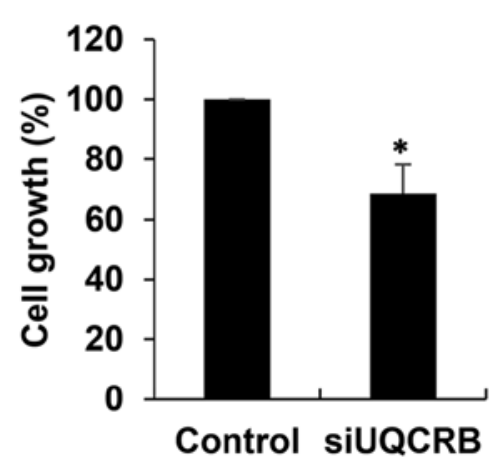

C
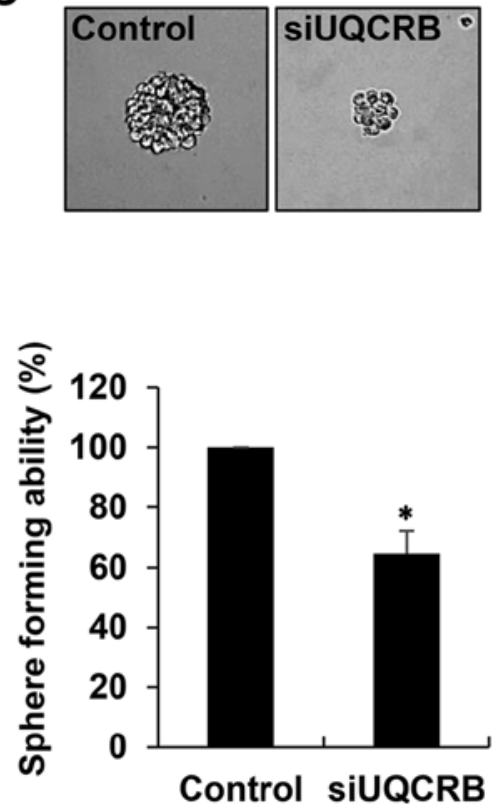

D
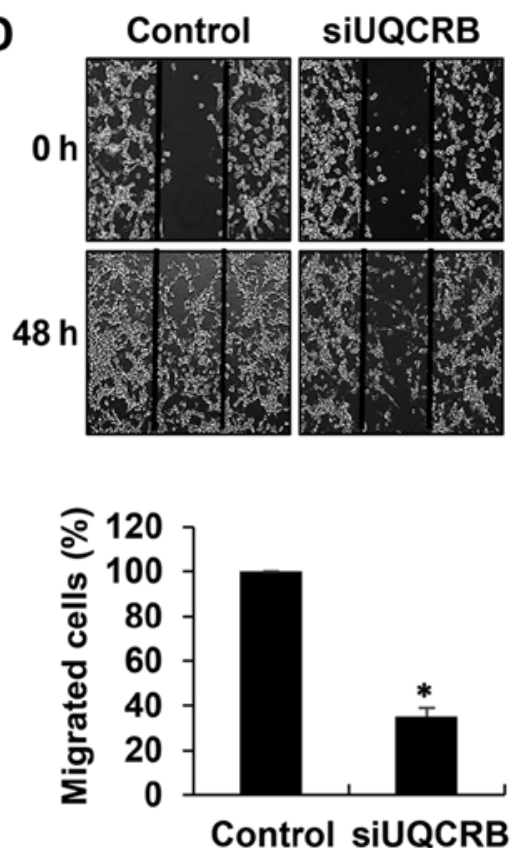

Figure 9. The effect of UQCRB knockdown on the cancer stem cell-like phenotypes of GBM cells. For the depletion of UQCRB mRNA, U87MG GSCs were transfected with either scrambled negative siRNA or UQCRB siRNA (siUQCRB). (A) Reverse transcription-PCR analysis of the knockdown of the UQCRB gene. (B-D) The effect of UQCRB knockdown on the growth (B), neurosphere formation (C) and migration of U87MG GSCs (D). " $\mathrm{P}<0.05$ versus the control.

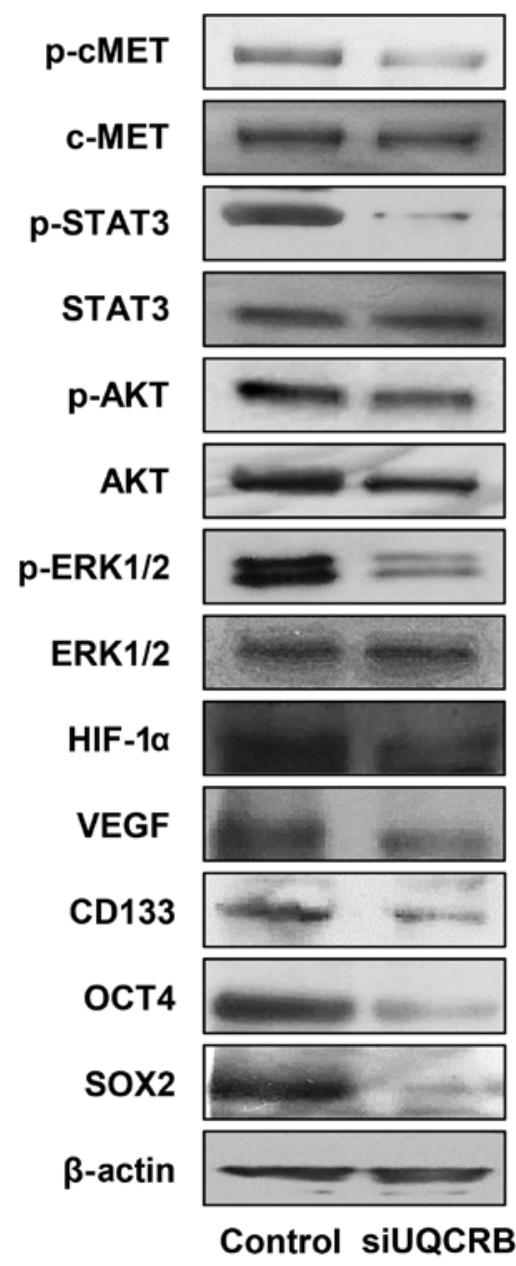

Figure 10. The effect of UQCRB knockdown on the c-Met signaling, HIF activity and stemness markers in U87MG GSCs. The protein levels were detected by western blot analysis using specific antibodies. The levels of $\beta$-actin were used as an internal control.
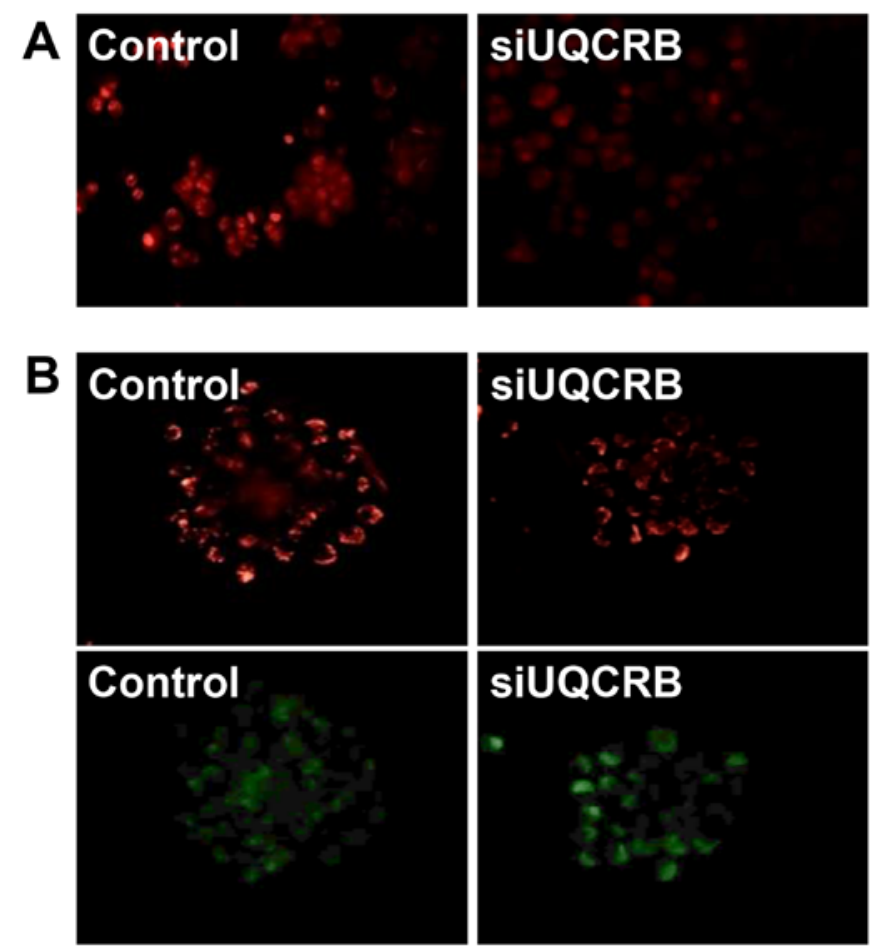

Figure 11. The effect of UQCRB knockdown on the mitochondrial function in U87MG GSCs. (A) The mitochondrial ROS generation was detected by MitoSOX $^{\mathrm{TM}}$ Red fluorescence using a fluorescence microscope. (B) The mitochondrial membrane potential was analyzed using the JC-1 fluorescent marker.

UQCRB knockdown significantly inhibited the growth of U87MG GSCs. In addition, the neurosphere formation and migration abilities of the GSCs were noteworthily suppressed by UQCRB knockdown (Fig. 9C and D). The results suggest 


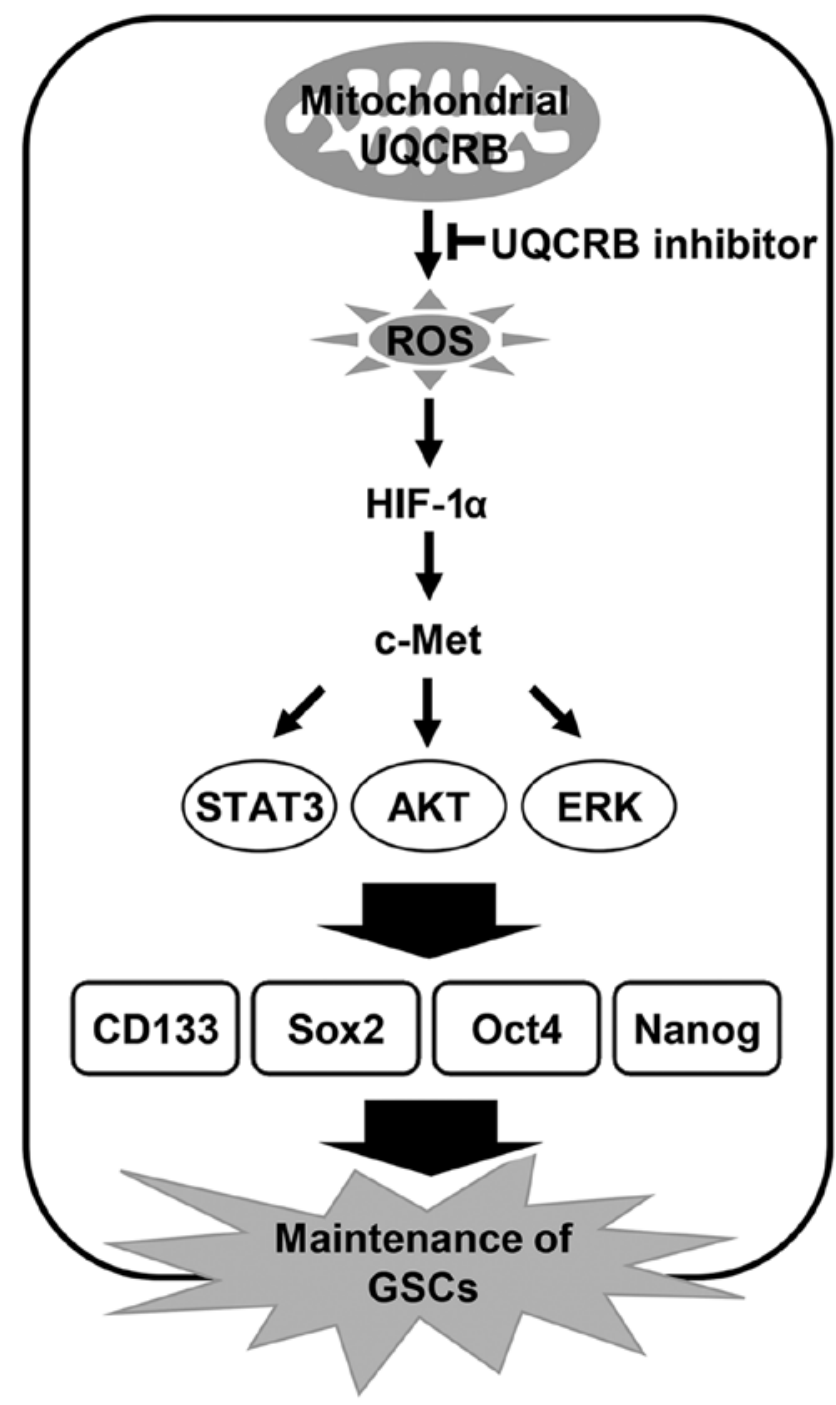

Figure 12. Proposed action mechanism of UQCRB inhibitors to eliminate GSCs. UQCRB inhibitors may suppress the maintenance of GSCs by downregulating mitochondrial ROS/HIF-1 $\alpha / \mathrm{c}-$ Met pathway.

that mitochondrial UQCRB positively regulates the cancer stem cell-like properties of GBM cells.

We next investigated the effect of UQCRB knockdown on the c-Met signaling, HIF and stemness markers in U87MG GSCs. UQCRB depletion reduced the activation of c-Met and its downstream signal transduction mediators, STAT3, Akt and ERK1/2 (Fig. 10). UQCRB knockdown also decreased the expression of HIF-1 $\alpha$ and VEGF. The downregulation of c-Met signaling and HIF activity consequently led to a reduction in the expression of GBM stemness markers such as CD133, Oct4 and Sox2 (Fig. 10).

Furthermore, we assessed whether the inhibition of the GSC characteristics by UQCRB depletion is associated with the regulation of mitochondrial function. UQCRB knockdown significantly suppressed the mitochondrial ROS generation and diminished the mitochondrial membrane potential in U87MG GSCs (Fig. 11). These results strongly demonstrate that UQCRB might function as a crucial mediator of GSC maintenance through the modulation of mitochondrial ROS generation in GSCs.

\section{Discussion}

Glioblastoma (GBM) is the most common and aggressive primary brain tumor in adults with poor prognosis (1-3). GBM is highly heterogeneous in nature and contains a small but highly tumorigenic and self-renewing population of cancer stem cells (6). Glioblastoma stem cells (GSCs) have been shown to contribute to tumor propagation and resistance to current therapeutic modalities $(7,8)$. Recent studies of human GBM have elucidated the genetic alterations common in this tumor, but much remains unknown about specific signaling pathways that regulate GSCs $(29,30)$. The activated c-Met receptor tyrosine kinase is known to stimulate cell survival, proliferation and invasion by triggering the activation of its multiple downstream effectors such as mitogen-activated protein kinases, Akt and STAT3 $(31,32)$. The overexpression of c-Met has been observed in aggressive human cancers including GBM and associated with poor prognosis in cancer patients $(33,34)$. In particular, activation of c-Met signaling led to an increase in expression of the stemness transcriptional regulators, Sox 2, Klf4, c-Myc, Oct4 and Nanog (22). Pharmacological inhibition of c-Met activity in GSCs prevented the activation of Oct4, Nanog and Klf4 and potently abrogated the clonogenicity, tumorigenicity and radioresistance of GSCs both in vitro and in vivo $(23,35)$. Therefore, therapeutic agents that block c-Met signaling pathway of GSCs would be an attractive candidate for the combination with current standard therapy against GBM.

In recent studies, the ubiquinol-cytochrome $c$ reductase binding protein (UQCRB) of mitochondrial complex III has been found to be a crucial mediator of mitochondrial ROS generation, thereby playing a central role in tumor angiogenesis via upregulation of both hypoxic and VEGF signaling $(12,13)$. In addition, small molecules targeting UQCRB resulted in the inhibition of mitochondrial ROS generation, and such inhibition consequently blocked hypoxiainducible factor (HIF) activation, vascular endothelial growth factor receptor 2 (VEGFR2) signal transduction and tumor angiogenesis in vivo $(14,15,36)$. Therefore, UQCRB has begun to emerge as a promising therapeutic target for antiangiogenic and anticancer therapy.

It has been recently reported that HIF-1 induces the overexpression and constitutive activation of c-Met (37). Furthermore, in melanoma cells, HIF-1 $\alpha$ stabilization by mitochondrial ROS promoted the c-Met-dependent prometastatic effects, such as enhancement of spreading on extracellular matrix, motility and invasion, as well as growth of metastatic colonies and the ability to form capillary-like structures by vasculogenic mimicry (38). Recent data revealed the involvement of ROS in the activation of the epithelial-mesenchymal transition (EMT) which is related to the acquisition and maintenance of stem cell-like characteristics, in several cancer models $(39,40)$. Taken together, mitochondrial UQCRB may be an upstream molecular target to control activation of ROS/HIF/c-Met axis and thus UQCRB inhibitors could be a new therapeutic agent to eradicate cancer stem cells.

The present study indicates that UQCRB inhibitors suppress cancer stem cell-like phenotypes in human glioblastoma cells by downregulating mitochondrial ROS/HIF-1 $\alpha / \mathrm{c}-\mathrm{Met}$ pathway (Fig. 12). Moreover, knockdown of UQCRB gene 
significantly inhibited the proliferation, neurosphere formation and migration of GSCs, as well as mitochondrial ROS generation, HIF-1 $\alpha$ accumulation, c-Met signaling activation and GSC stemness marker expression. In conclusion, these findings suggest that targeting UQCRB function associated with regulation of mROS generation could be an attractive therapeutic strategy for interrupting the c-Met signaling that is important for GSC maintenance. Accordingly, our results provide new insight into the role of mitochondrial UQCRB in the activation of c-Met signaling of GSCs and the underlying mechanism of UQCRB inhibitors to eliminate GSCs.

\section{Acknowledgements}

This study was supported by 'Cooperative Research Program for Agriculture Science and Technology Development (Project no. PJ01188001)' Rural Development Administration, Republic of Korea, the Basic Science Research Program through the National Research Foundation of Korea (NRF) funded by the Ministry of Education (NRF-2016R1D1A1B03932956), and the Brain Korea 21 Plus Project, Republic of Korea. This study was also supported in part by NRF grant 2012M3A9D1054520 and 2015K1A1A2028365.

\section{References}

1. Louis DN, Perry A, Reifenberger G, von Deimling A, Figarella-Branger D, Cavenee WK, Ohgaki H, Wiestler OD, Kleihues P and Ellison DW: The 2016 World Health Organization Classification of Tumors of the Central Nervous System: A summary. Acta Neuropathol 131: 803-820, 2016

2. Lutterbach J, Guttenberger R and Pagenstecher A: Gliosarcoma: A clinical study. Radiother Oncol 61: 57-64, 2001.

3. Wen PY and Kesari S: Malignant gliomas in adults. N Engl J Med 359: 492-507, 2008

4. Stupp R, Mason WP, van den Bent MJ, Weller M, Fisher B, Taphoorn MJ, Belanger K, Brandes AA, Marosi C, Bogdahn U, et al; European Organisation for Research and Treatment of Cancer Brain Tumor and Radiotherapy Groups; National Cancer Institute of Canada Clinical Trials Group: Radiotherapy plus concomitant and adjuvant temozolomide for glioblastoma. N Engl J Med 352: 987-996, 2005.

5. Stavrovskaya AA, Shushanov SS and Rybalkina EY: Problems of glioblastoma multiforme drug resistance. Biochemistry (Mosc) 81: 91-100, 2016.

6. Sundar SJ, Hsieh JK, Manjila S, Lathia JD and Sloan A: The role of cancer stem cells in glioblastoma. Neurosurg Focus 37: E6, 2014.

7. Bao S, Wu Q, McLendon RE, Hao Y, Shi Q, Hjelmeland AB, Dewhirst MW, Bigner DD and Rich JN: Glioma stem cells promote radioresistance by preferential activation of the DNA damage response. Nature 444: 756-760, 2006.

8. Liu G, Yuan X, Zeng Z, Tunici P, Ng H, Abdulkadir IR, Lu L, Irvin D, Black KL and Yu JS: Analysis of gene expression and chemoresistance of $\mathrm{CD}_{133^{+}}$cancer stem cells in glioblastoma. Mol Cancer 5: 67, 2006.

9. Cho DY, Lin SZ, Yang WK, Lee HC, Hsu DM, Lin HL, Chen CC, Liu CL, Lee WY and Ho LH: Targeting cancer stem cells for treatment of glioblastoma multiforme. Cell Transplant 22: 731-739, 2013.

10. Kalkan R: Glioblastoma stem cells as a new therapeutic target for glioblastoma. Clin Med Insights Oncol 9: 95-103, 2015.

11. Jung HJ, Lee HB, Kim CJ, Rho JR, Shin J and Kwon HJ: Anti-angiogenic activity of terpestacin, a bicyclo sesterterpene from Embellisia chlamydospora. J Antibiot (Tokyo) 56: 492-496, 2003.

12. Jung HJ, Shim JS, Lee J, Song YM, Park KC, Choi SH, Kim ND, Yoon JH, Mungai PT, Schumacker PT, et al: Terpestacin inhibits tumor angiogenesis by targeting UQCRB of mitochondrial complex III and suppressing hypoxia-induced reactive oxygen species production and cellular oxygen sensing. J Biol Chem 285: 11584-11595, 2010.
13. Jung HJ, Kim Y, Chang J, Kang SW, Kim JH and Kwon HJ: Mitochondrial UQCRB regulates VEGFR2 signaling in endothelial cells. J Mol Med (Berl) 91: 1117-1128, 2013.

14. Jung HJ, Kim KH, Kim ND, Han G and Kwon HJ: Identification of a novel small molecule targeting UQCRB of mitochondrial complex III and its anti-angiogenic activity. Bioorg Med Chem Lett 21: 1052-1056, 2011.

15. Jung HJ, Cho M, Kim Y, Han G and Kwon HJ: Development of a novel class of mitochondrial ubiquinol-cy tochrome $c$ reductase binding protein (UQCRB) modulators as promising antiangiogenic leads. J Med Chem 57: 7990-7998, 2014.

16. Galli R, Binda E, Orfanelli U, Cipelletti B, Gritti A, De Vitis S, Fiocco R, Foroni C, Dimeco F and Vescovi A: Isolation and characterization of tumorigenic, stem-like neural precursors from human glioblastoma. Cancer Res 64: 7011-7021, 2004.

17. Laks DR, Masterman-Smith M, Visnyei K, Angenieux B, Orozco NM, Foran I, Yong WH, Vinters HV, Liau LM, Lazareff JA, et al: Neurosphere formation is an independent predictor of clinical outcome in malignant glioma. Stem Cells 27: 980-987, 2009.

18. Lee J, Kotliarova S, Kotliarov Y, Li A, Su Q, Donin NM, Pastorino S, Purow BW, Christopher N, Zhang W, et al: Tumor stem cells derived from glioblastomas cultured in bFGF and EGF more closely mirror the phenotype and genotype of primary tumors than do serum-cultured cell lines. Cancer Cell 9: 391-403, 2006.

19. Singh SK, Hawkins C, Clarke ID, Squire JA, Bayani J, Hide T, Henkelman RM, Cusimano MD and Dirks PB: Identification of human brain tumour initiating cells. Nature 432: 396-401, 2004.

20. Cheng L, Wu Q, Guryanova OA, Huang Z, Huang Q, Rich JN and Bao S: Elevated invasive potential of glioblastoma stem cells. Biochem Biophys Res Commun 406: 643-648, 2011.

21. Zhang X, Zhang W, Mao XG, Zhen HN, Cao WD and Hu SJ: Targeting role of glioma stem cells for glioblastoma multiforme. Curr Med Chem 20: 1974-1984, 2013.

22. Li Y, Li A, Glas M, Lal B, Ying M, Sang Y, Xia S, Trageser D, Guerrero-Cázares H, Eberhart CG, et al: c-Met signaling induces a reprogramming network and supports the glioblastoma stem-like phenotype. Proc Natl Acad Sci USA 108: 9951-9956, 2011.

23. Joo KM, Jin J, Kim E, Ho Kim K, Kim Y, Gu Kang B, Kang YJ, Lathia JD, Cheong KH, Song PH, et al: MET signaling regulates glioblastoma stem cells. Cancer Res 72: 3828-3838, 2012.

24. Keith B and Simon MC: Hypoxia-inducible factors, stem cells, and cancer. Cell 129: 465-472, 2007.

25. Qiang L, Wu T, Zhang HW, Lu N, Hu R, Wang YJ, Zhao L, Chen FH, Wang XT, You QD, et al: HIF-1 $\alpha$ is critical for hypoxiamediated maintenance of glioblastoma stem cells by activating Notch signaling pathway. Cell Death Differ 19: 284-294, 2012.

26. Shi X, Zhang Y, Zheng J and Pan J: Reactive oxygen species in cancer stem cells. Antioxid Redox Signal 16: 1215-1228, 2012.

27. Ding S, Li C, Cheng N, Cui X, Xu X and Zhou G: Redox regulation in cancer stem cells. Oxid Med Cell Longev 2015: 750798, 2015.

28. Im CN, Yun HH, Yoo HJ, Park MJ and Lee JH: Enhancement of SOX-2 expression and ROS accumulation by culture of A172 glioblastoma cells under non-adherent culture conditions. Oncol Rep 34: 920-928, 2015.

29. Haque A, Banik NL and Ray SK: Molecular alterations in glioblastoma: Potential targets for immunotherapy. Prog Mol Biol Transl Sci 98: 187-234, 2011.

30. Ohgaki $\mathrm{H}$ and Kleihues P: Genetic pathways to primary and secondary glioblastoma. Am J Pathol 170: 1445-1453, 2007.

31. Birchmeier C, Birchmeier W, Gherardi E and Vande Woude GF: Met, metastasis, motility and more. Nat Rev Mol Cell Biol 4: 915-925, 2003.

32. Trusolino L, Bertotti A and Comoglio PM: MET signalling: Principles and functions in development, organ regeneration and cancer. Nat Rev Mol Cell Biol 11: 834-848, 2010.

33. Kong DS, Song SY, Kim DH, Joo KM, Yoo JS, Koh JS, Dong SM, Suh YL, Lee JI, Park K, et al: Prognostic significance of c-Met expression in glioblastomas. Cancer 115: 140-148, 2009.

34. Nabeshima K, Shimao Y, Sato S, Kataoka H, Moriyama T, Kawano H, Wakisaka S and Koono M: Expression of c-Met correlates with grade of malignancy in human astrocytic tumours: An immunohistochemical study. Histopathology 31: 436-443, 1997.

35. Kim B, Jung N, Lee S, Sohng JK and Jung HJ: Apigenin inhibits cancer stem cell-like phenotypes in human glioblastoma cells via suppression of c-Met signaling. Phytother Res 30: 1833-1840, 2016. 
36. Jung $\mathrm{HJ}$ and Kwon HJ: Exploring the role of mitochondrial UQCRB in angiogenesis using small molecules. Mol Biosyst 9: 930-939, 2013.

37. Pennacchietti S, Michieli P, Galluzzo M, Mazzone M, Giordano S and Comoglio PM: Hypoxia promotes invasive growth by transcriptional activation of the met protooncogene. Cancer Cell 3: 347-361, 2003.

38. Comito G, Calvani M, Giannoni E, Bianchini F, Calorini L, Torre E, Migliore C, Giordano S and Chiarugi P: HIF-1 $\alpha$ stabilization by mitochondrial ROS promotes Met-dependent invasive growth and vasculogenic mimicry in melanoma cells. Free Radic Biol Med 51: 893-904, 2011.
39. Radisky DC, Levy DD, Littlepage LE, Liu H, Nelson CM, Fata JE, Leake D, Godden EL, Albertson DG, Nieto MA, et al: Raclb and reactive oxygen species mediate MMP-3-induced EMT and genomic instability. Nature 436: 123-127, 2005.

40. Cannito S, Novo E, Compagnone A, Valfrè di Bonzo L, Busletta C, Zamara E, Paternostro C, Povero D, Bandino A, Bozzo F, et al: Redox mechanisms switch on hypoxia-dependent epithelial-mesenchymal transition in cancer cells. Carcinogenesis 29: 2267-2278, 2008. 